\title{
Identification and analysis of serum samples by surface-enhanced Raman spectroscopy combined with characteristic ratio method and PCA for gastric cancer detection
}

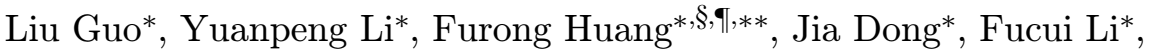 \\ Xinhao Yang*, Siqi Zhu* and Maoxun Yang ${ }^{\dagger, *} \|, * *$ \\ *Department of Opto-Electronic Engineering \\ Jinan University, Guangzhou 510632, P. R. China \\ ${ }^{\dagger}$ Zhuhai Da Hengqin Science and Technology Development Co., Ltd \\ Hengqin New Area, Zhuhai 519000, P. R. China \\ $\$$ Zhuhai Hopegenes Medical \& Pharmaceutical Institute Co., Ltd \\ Hengqin New Area, Zhuhai 519000, P. R. China \\ ${ }^{\S}$ Research Institute of Jinan University in Dongguan \\ Dongguan 523000, P. R. China \\ Ifurong_huang@163.com \\ "yangmaoxun1980@163.com
}

Received 11 July 2018

Accepted 10 December 2018

Published 10 January 2019

\begin{abstract}
This study aimed to explore the application of surface-enhanced Raman scattering (SERS) in the rapid diagnosis of gastric cancer. The SERS spectra of 68 serum samples from gastric cancer patients and healthy volunteers were acquired. The characteristic ratio method (CRM) and principal component analysis (PCA) were used to differentiate gastric cancer serum from normal serum. Compared with healthy volunteers, the serum SERS intensity of gastric cancer patients was relatively high at $722 \mathrm{~cm}^{-1}$, while it was relatively low at 588, 644, 861, 1008, 1235, 1397, 1445 and $1586 \mathrm{~cm}^{-1}$. These results indicated that the relative content of nucleic acids in the serum of gastric cancer patients rises while the relative content of amino acids and carbohydrates decreases. In PCA, the sensitivity and specificity of discriminating gastric cancer were $94.1 \%$ and $94.1 \%$, respectively, with the accuracy of $94.1 \%$. Based on the intensity ratios of four characteristic peaks at $722,861,1008$ and $1397 \mathrm{~cm}^{-1}, \mathrm{CRM}$ presented the diagnostic sensitivity and specificity of $100 \%$ and $97.4 \%$, respectively, and the accuracy of $98.5 \%$. Therefore, the three peak intensity ratios of $\mathrm{I}_{722} / \mathrm{I}_{861}, \mathrm{I}_{722} / \mathrm{I}_{1008}$ and $\mathrm{I}_{722} / \mathrm{I}_{1397}$ can be considered as biological fingerprint
\end{abstract}

\footnotetext{
${ }^{* *}$ Corresponding authors.
}

This is an Open Access article published by World Scientific Publishing Company. It is distributed under the terms of the Creative Commons Attribution 4.0 (CC-BY) License. Further distribution of this work is permitted, provided the original work is properly cited. 
information for gastric cancer diagnosis and can rapidly and directly reflect the physiological and pathological changes associated with gastric cancer development. This study provides an important basis and standards for the early diagnosis of gastric cancer.

Keywords: Surface-enhanced Raman spectroscopy; serum; gastric cancer; characteristic ratio method; principal components analysis.

\section{Introduction}

Gastric cancer is currently the fourth most common malignancy and the second leading cause of cancerrelated deaths worldwide. Approximately 650,000 individuals have died from this type of cancer, and 880,000 new cases of gastric cancer are diagnosed each year. Improving the diagnosis of gastric cancer is the most important approach to reducing the mortality rate of this malignancy. ${ }^{1,2}$ Endoscopy combined with histopathological analysis is the most accurate method for the diagnosis of gastric cancer. This method, however, inflicts damage on patients. In addition, the subjectivity of pathological diagnosis and the uncertainty of endoscopic positioning are unavoidable and often result in the misdiagnosis of small lesions in the early stage of gastric cancer. ${ }^{3-5}$ Therefore, identifying a highly sensitive, rapid, and noninvasive screening method for the diagnosis of gastric cancer is vital. ${ }^{6-8}$

Near-infrared Raman spectroscopy can be used to monitor the changes associated with the development of diseases because it reflects differential changes in biomolecules, such as proteins, carbohydrates and lipids. ${ }^{9,10}$ Surface-enhanced Raman scattering (SERS) spectra has considerable potential as a cancer detection method given its high specificity and sensitivity. ${ }^{11-13}$ In the field of biomedicine, in recent years, SERS technique has shown to be an excellent technique for the biopsybased and body-fluid-based detection of some cancers such as gastric cancer, breast cancer, colorectal cancer, esophagus cancer and cervical cancers. ${ }^{14-20}$ It has demonstrated good application value in the body-fluid-based detection of cancer and is expected to provide new diagnostic criteria for the clinical detection and study of cancer. ${ }^{21}$ Among the body fluids detection, the applications of blood detection are the most frequent. ${ }^{22}$ Many research teams have attempted to utilize the SERS technique in bloodbased cancer detection. Feng et al. used a polarized laser to acquire the plasma SERS spectra of healthy volunteers and gastric cancer patients, and combined the multivariate statistical methods of principal component analysis (PCA) and linear discriminant analysis (LDA) to distinguish the blood samples of healthy volunteers from those of cancer patients. The diagnostic sensitivity and specificity were $79.5 \%$ and $91 \%$, respectively. The results proved that the healthy volunteers and the gastric cancer patients can be differentiated by the SERS spectra of human plasma. ${ }^{23}$ Vargas-Obieta et al. collected the SERS spectra of the serum samples of 14 controls and 14 breast cancer patients and demonstrated that SERS and PCA-LDA can be used to discriminate between control and cancer samples with $96 \%$ sensitivity and $87 \%$ specificity. ${ }^{24}$ Sánchez-Rojo et al. collected serum SERS spectra of 14 controls and 14 cervical cancer patients. The spectral data was processed using multivariate statistical analysis including principal component analysis (PCA) and LDA, and the sensitivity and specificity for correctly classifying control and cervical cancer samples were both $88 \% .^{25}$

PCA and LDA are the main mathematical statistical methods used for cancer detection based on Raman spectroscopy. ${ }^{26}$ Given the complexity of multidimensional analyses and the massive size of spectral data, a simple and accurate multivariate statistical method for the rapid detection of cancer in body fluid must be developed on the basis of the SERS technique. ${ }^{27}$ The characteristic ratio method $(\mathrm{CRM})$ is a popular unsupervised statistical analysis method that discriminates samples by correlating variations in sample spectra with sample pathology on the basis of peak intensity ratios. CRM is simpler and faster than PCA. Thus, a diagnostic method that combines SERS with CRM may have potential clinical applications in the diagnosis of gastric cancer.

In this study, serum samples collected from 34 healthy volunteers and 34 patients who had been diagnosed with gastric cancer through histopathological methods were used as the research objects. Gold nanoparticles were used as the enhancement 
base. The differences in SERS spectra between gastric cancer patients and healthy volunteers were compared to study the changes in serum substance concentrations. Finally, the CRM and PCA were used to distinguish the SERS spectra of the serum samples of normal and gastric cancer patients for the rapid diagnosis of gastric cancer.

\section{Experimental Materials and Methods}

\subsection{Preparation and characterization of gold colloids}

Gold colloids were prepared by using sodium citrate to restore chloroauric acid. A total of $100 \mathrm{ml}$ of $0.01 \%$ chloroauric acid solution was boiled on a heated magnetic stirring apparatus. The solution was then removed from the stirring apparatus, mixed with $1 \mathrm{ml}$ of $1 \%$ sodium citrate, then stirred and heated for $15 \mathrm{~min}$. A wine-red gold sol solution was obtained after intense boiling and cooling. The solution was centrifuged for further use. The gold sol was dropped to the microscope slide and dried for further measurement.

Figure 1 showed the extinction spectrum and the image of scanning electron microscope of the gold sol prepared in the trial. It was measured with ultraviolet and visible spectrophotometer (supplied by Shimadzu Corporation, with model of UV-2550), and the absorption peak of the gold sol was located at $520 \mathrm{~nm}$, and its measured spectral bandwidth from 300 to $900 \mathrm{~nm}$. The gold sol was further measured using the field emission scanning electron microscope (supplied by German Zeiss, with the

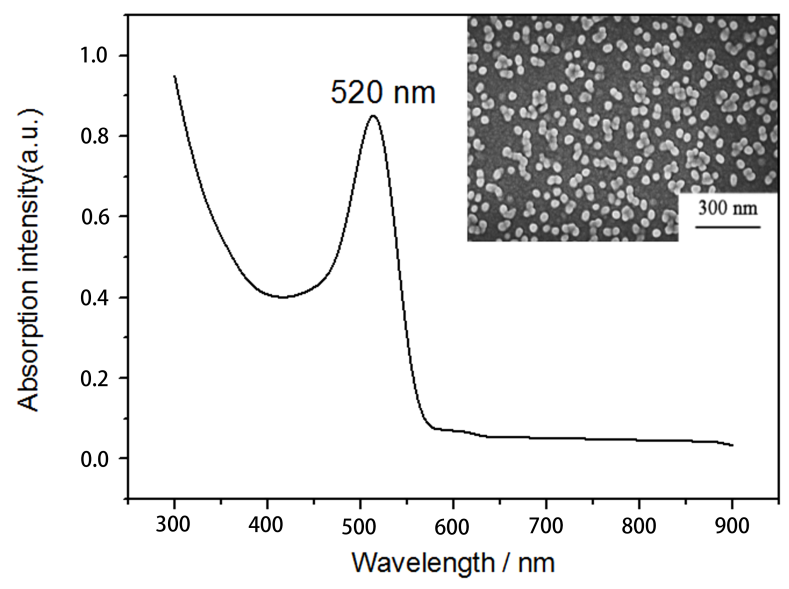

Fig. 1. The UV/Visible absorption spectrum of the Au colloid. The absorption maximum is located at $520 \mathrm{~nm}$. The inserted picture is the the TEM micrograph of the Au nanoparticles. model of ultra55), which showed that the average diameter of the gold sol was of $40 \pm 3 \mathrm{~nm}$.

\subsection{Preparation of human serum samples}

A total of 68 serum samples were collected from the patients of The First Affiliated Hospital of Jinan University. Among these samples, 34 were collected from patients who were newly diagnosed with gastric cancer, and 34 were collected from healthy volunteers. The mean age of patients with gastric cancer was $53 \pm 10$ years, whereas that of healthy volunteers was $48 \pm 12$ years. All samples were collected from patients with the same racial and socioeconomic backgrounds. Serum were mixed with gold colloid suspension at the volume ratio of $1: 2$, maintained at $4^{\circ} \mathrm{C}$ for $2 \mathrm{~h}$, and then transferred to a cuvette for SERS detection.

\subsection{Raman instrumentation}

A portable Raman spectrometer was used as the measurement instrument. The measurement parameters were as follows: excitation wavelength of $785 \mathrm{~nm}$, power of $70 \mathrm{~mW}$, spectral resolution of $8 \mathrm{~cm}^{-1}$, and spectral window of $400-1800 \mathrm{~cm}^{-1}$.

Given that the acquired Raman spectra exhibited baseline drift and measurement was affected by random noise, the $400-1800 \mathrm{~cm}^{-1}$ region in Raman spectra was subjected to background subtraction and smoothing using Origin 8.5 software.

\section{Results and Discussion}

\subsection{Analysis of serum surface- enhanced Raman scattering (SERS) spectra}

The averaged conventional Raman spectra of serum samples from 34 healthy volunteers and 34 gastric cancer patients are shown in Fig. 2. The Raman spectra of the serum were collected at integration times of 10,20, 30 and $60 \mathrm{~s}$. It can be seen that no obvious Raman signal is detected in both normal and gastric cancer serum without using gold nanoparticles. The Raman signals are negligibly enhanced with the prolongation of integration time. A slight baseline drift is observed at the integration time of $60 \mathrm{~s}$. 


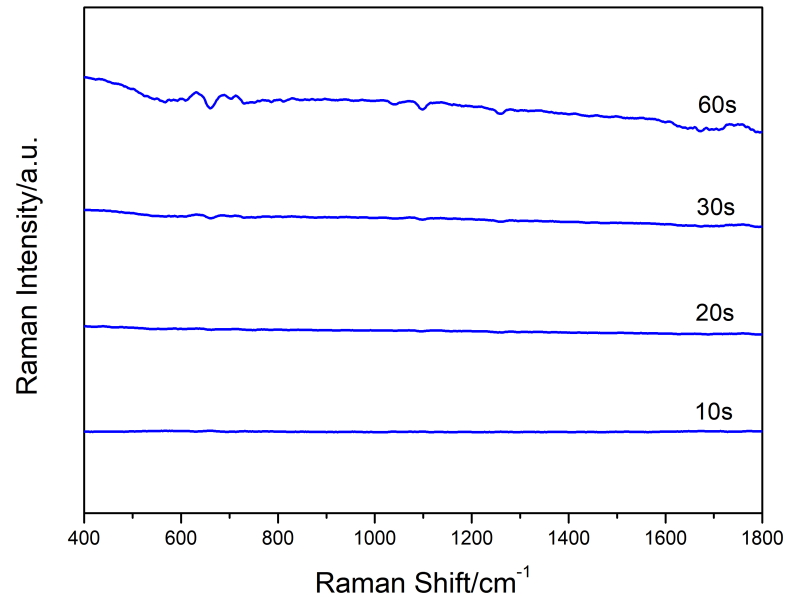

(a)

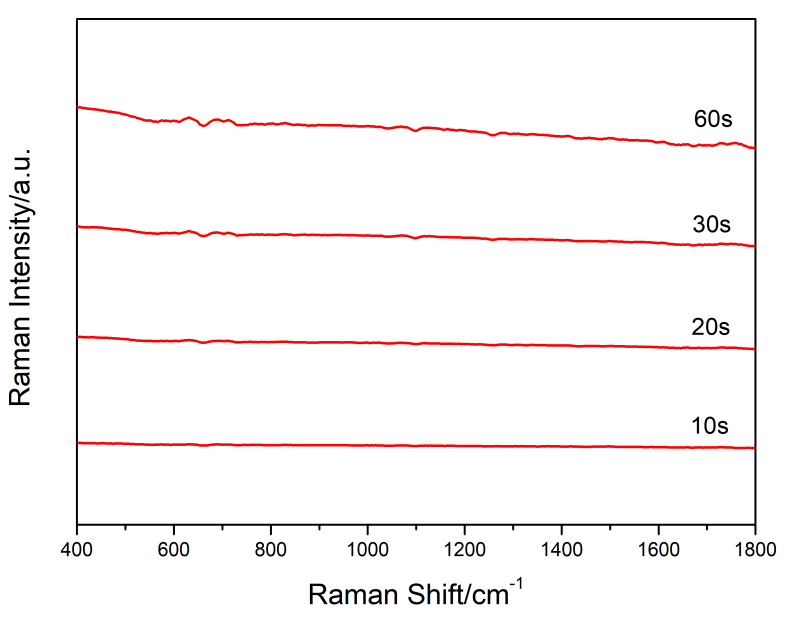

(b)

Fig. 2. The averaged conventional Raman spectra of serum samples: (a) healthy volunteers; (b) gastric cancer patients.

Figure 3 shows the comparison of the mean SERS spectra between 34 healthy volunteers and 34 gastric cancer patients, and the integration time for all samples is $10 \mathrm{~s}$. It can be seen that the SERS spectra are considerably amplified relative to conventional Raman spectra. The inset in the upper portion of Fig. 3 shows the mean SERS spectra of serum of 34 healthy volunteers and 34 gastric cancer patients, the shaded areas represent the differences among individuals in the same group. The SERS spectra of both groups show good repeatability. The SERS spectra of both groups show intense peaks at 505 (attributable to isoleucine), 588 (attributable to ascorbic acid and amino

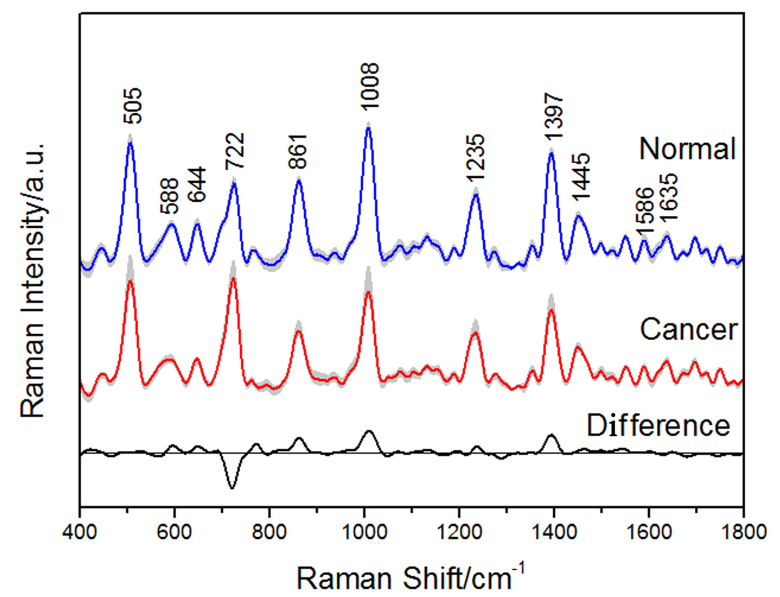

Fig. 3. Comparison of the mean SERS spectra for the normal serum $(n=34)$ vs that of the gastric cancer $(n=34)$. The shaded areas represent the standard deviations of SERS spectra of normal and cancer serum. The bottom spectrum corresponds to the difference of normal and cancer spectra. compound), 644 (attributable to tyrosine), 722 (attributable to hypoxanthine), 861 (attributable to tryptophan), 1008 (attributable to phenylalanine), 1235 (attributable to D-mannose), 1397 (attributable to tryptophan), 1445 (attributable to elastin and collagen), 1586 (attributable to phenylalanine) and $1635 \mathrm{~cm}^{-1}$ (attributable to leucine). In order to better understand the biochemical information reflected in the serum SERS spectra at the molecular level, the main bands observed in the normal and gastric cancer SERS spectra and the corresponding assignment of biomolecules are listed in Table $1 .{ }^{28-30}$

The lower part of Fig. 3 shows the difference spectrum of the mean SERS spectra between healthy volunteers and gastric cancer patients, consider the biochemical differences among healthy volunteers, the Student's $t$-test was performed to ensure that the observed differences of peak intensity correspond effectively between normal and cancer patients. Nine SERS peaks with statistical significance $(p<0.05)$ were selected for analysis, that is $588,644,722,861,1008,1235,1397,1445$ and $1586 \mathrm{~cm}^{-1}$. Comparing the intensities of these nine characteristic peaks reveals that the peak at $722 \mathrm{~cm}^{-1}$ in serum spectra of gastric cancer patients has high intensity. This peak is assigned to the inplane bending vibration mode of the $\mathrm{C}-\mathrm{H}$ bond of adenine in nucleic acid bases. This finding shows that the serum nucleic acid content of gastric cancer patients is higher than that of the healthy volunteers. The intensities of the characteristic peaks at $588,644,861,1008,1235,1397,1445$ and $1586 \mathrm{~cm}^{-1}$ 
Table 1. SERS peak positions and vibrational mode assignments and the comparison of the intensity of mean SERS spectra of normal and cancer $\left(10^{-3}\right.$, mean \pm s.d. $)$.

\begin{tabular}{lclrrr}
\hline Raman peak $\left(\mathrm{cm}^{-1}\right)$ & Vibrational mode & Major assignment & Normal & \multicolumn{1}{c}{ Cancer } & $P$ \\
\hline 505 & Ring vibration & L-arginine & $17.31 \pm 6.68$ & $17.30 \pm 7.02$ & 0.0726 \\
588 & & Ascorbic acid amide-VI & $5.09 \pm 0.81$ & $3.93 \pm 0.97$ & 0.0264 \\
644 & $\nu(\mathrm{C}-\mathrm{S})$ & Tyrosine & $5.54 \pm 0.57$ & $4.60 \pm 0.71$ & 0.0301 \\
722 & $\nu(\mathrm{C}-\mathrm{H})$ & hypoxanthine & $11.93 \pm 2.84$ & $17.32 \pm 3.14$ & 0.0164 \\
861 & $\delta(\mathrm{C}-\mathrm{O}-\mathrm{H})$ & L-tryptophan & $11.59 \pm 0.46$ & $8.98 \pm 0.92$ & 0.0203 \\
1008 & $\nu \mathrm{s}(\mathrm{C}-\mathrm{C})$ & L-phenylalanine & $16.86 \pm 3.77$ & $12.99 \pm 3.95$ & 0.0189 \\
1235 & $\nu(\mathrm{C}-\mathrm{N})$ & D-mannos & $10.42 \pm 0.22$ & $7.14 \pm 0.43$ & 0.0311 \\
1397 & $\delta\left(\mathrm{CH}_{2}\right)$ & L-tryptophan & $12.58 \pm 1.66$ & $10.57 \pm 1.50$ & 0.0195 \\
1445 & $\delta(\mathrm{CH})$ & Collagen, phospholipids & $6.56 \pm 0.09$ & $5.55 \pm 0.11$ & 0.0445 \\
1586 & $\delta(\mathrm{C}=\mathrm{C})$ & Phenylalanine & $2.81 \pm 0.09$ & $2.77 \pm 0.07$ & 0.0451 \\
1635 & $\nu(\mathrm{C}=\mathrm{O})$ & L-Leucine & $5.42 \pm 0.04$ & $5.33 \pm 0.06$ & 0.0643 \\
\hline
\end{tabular}

Notes: $\nu$, stretching vibration; $\delta$, bending vibration; $\nu$ s, symmetric stretch.

in the serum spectra of gastric cancer patients are weaker than those of the same peaks in the serum spectra of healthy volunteers. The weak intensity of these peaks, which are attributable to carbohydrates and amino acids, indicates that the carbohydrate and amino acid contents of cancer patients are lower than those of healthy volunteers. The serum of patients with gastric cancer have elevated adenine contents and reduced protein contents because metabolism intensifies and large amounts of glucose and amino acids are consumed to provide the energy required by the excessive proliferation of cancer cells during carcinogenesis. The condition of abnormal metabolism is in line with the results of plasma detection using the SERS technique performed on nasopharynx cancer and gastric cancer by Feng et al.

Although the SERS spectra provided rich information, gastric cancer cannot be diagnosed accurately through the simple comparison of the characteristic peak intensities between healthy volunteers and gastric cancer patients. Therefore, it is necessary to further analyze the SERS spectral data by means of statistical methods to obtain better diagnosis results of gastric cancer.

\subsection{Analysis of SERS spectra of samples of gastric cancer patients and healthy volunteers}

\subsubsection{Principal component analysis}

Principle Component Analysis (PCA) is a multivariate statistical method commonly used to analyze the Raman spectra of different biological samples. Several principal components (PCs) with major contributions can be obtained by simplifying complicated spectroscopic data. These PCs characterize most spectral information and can be intuitively represented in two-dimensional and three-dimensional coordinates.

In this study, the polynomial fitting was used to subtract the fluorescent background of the original SERS spectral data. Then, PCA statistical analyses were performed on normalized spectroscopic data, and PC1, PC2, and PC4, the three PCs with the most significant differences, were selected through Student's $t$-test. The cumulative percentage variance of these three PCs is $83 \%$.

As shown in Fig. 4, the two-dimensional scatter plot is drawn using $\mathrm{PC} 1, \mathrm{PC} 2$ and $\mathrm{PC} 4$ scores. Based on the combination of different significant principal component, most of serum samples of gastric cancer and healthy volunteers are clustered in two different areas. Figure 4(a) shows the PC1 and $\mathrm{PC} 2$ scatter plots for cancer and normal group data with the corresponding discriminant line, $\mathrm{PC} 2=-2.11 \mathrm{PC} 1+0.51$, and so $91.2 \% \quad(31 / 34)$ sensitivity and $97.4 \%(33 / 34)$ specificity, with the accuracy of $94.1 \%$ (64/68); In addition, Fig. 4(b) shows the PC1 and PC4 scatter plot for cancer and normal group data with the corresponding discriminant lines, $\mathrm{PC} 4=2.83 \mathrm{PC} 1-0.91$, and so $91.2 \%$ $(31 / 34)$ sensitivity and $88.2 \%(30 / 34)$ specificity, with the accuracy of $89.7 \%(61 / 68)$. The result shows that the selection of different combinations of significant PCs will provide different levels of accuracy for serum classification. 


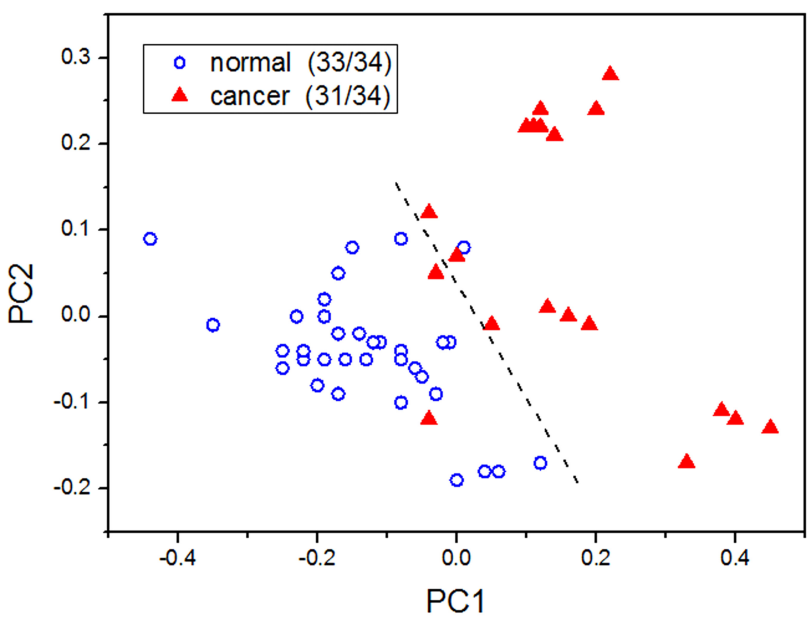

(a)

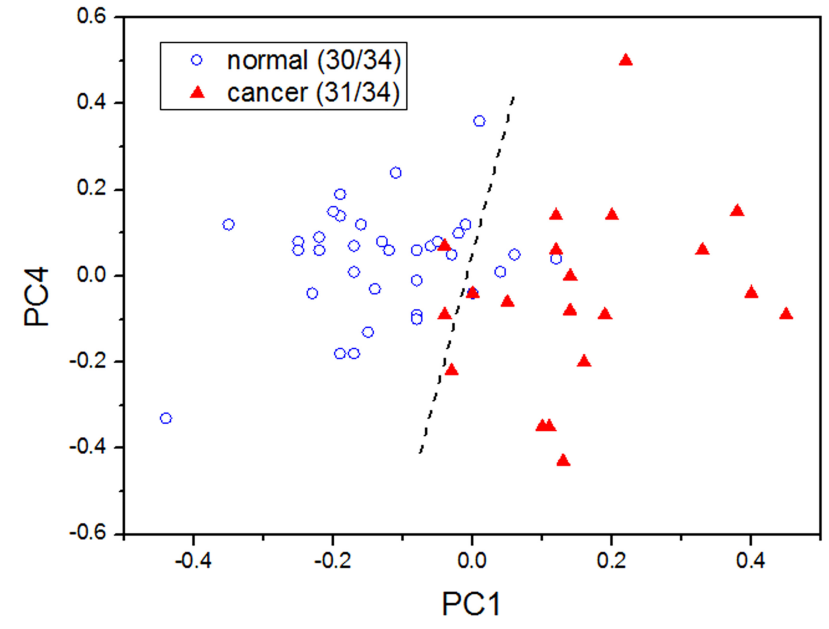

(b)

Fig. 4. Scatter plots of the principal component (PC) scores for normal and gastric cancer serum spectra: (a) PC1 vs PC2; (b) PC1 vs PC4.

As shown in Fig. 5, the three-dimensional scatter plot is drawn based on PC1, PC2, PC4, and the sensitivity and specificity are $94.1 \%(32 / 34)$ and $94.1 \%(32 / 34)$ respectively, with the accuracy rate of $94.1 \%(64 / 68)$. From Fig. 5, it is clear that, although there is partial overlapping between normal and gastric cancer group, the two groups can be easily distinguished. The spatial distribution of the cancer group is relatively scattered while the normal group is relatively concentrated. This distribution pattern may be attributed to the stable serum component levels of the healthy volunteers given their normal physiological state. By contrast, the

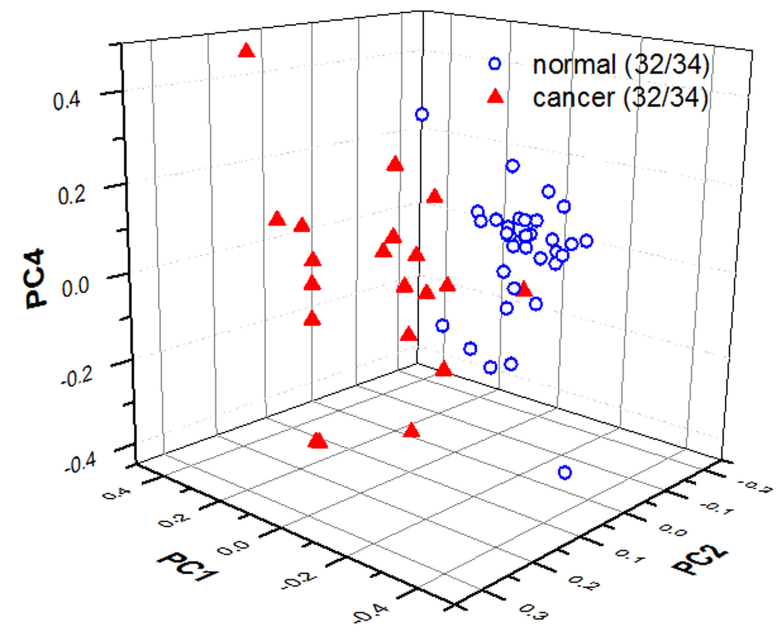

Fig. 5. (Color online) A three-dimensional scatter plot of the principal component (PC) scores of serum SERS spectra in gastric cancer (red triangle) and normal (blue circle) group. serum component levels of patients with cancer, who are in a pathological state, may drastically change. These results show that PCA can preliminarily discriminate the gastric cancer group from the normal group. Nevertheless, identifying the molecules that contribute to spectral differences is difficult, and the composition of human serum is complex. Therefore, PCA cannot be used to reflect negligible changes in the spectral shape and spectral intensity of serum SERS spectra.

\subsubsection{Characteristic ratio method}

SERS peaks attributable to different substances, such as proteins, fats, carbohydrates, and vitamins, reflect metabolic status and can be taken as important fingerprint peaks for cancer diagnosis. ${ }^{31}$ Diseases have been diagnosed by analyzing the intensities of Raman characteristic peaks or the intensity ratios of different peaks. For example, the Raman intensity ratios of peaks at 1455 and $1655 \mathrm{~cm}^{-1}$ have been used to differentiate the serum of healthy volunteers from those of breast cancer patients and normal tissue from the tissue of malignant cervix neoplasm. ${ }^{32,33}$ Lin et al. diagnosed colorectal adenocarcinomas by using the intensity ratio of SERS peaks at 725 and $638 \mathrm{~cm}^{-1}$, which are attributable to adenine and tyrosine, respectively, as biological fingerprint information.

This study also attempted to use the peak intensity ratios of serum SERS spectra for the rapid diagnosis of gastric cancer. The four characteristic 


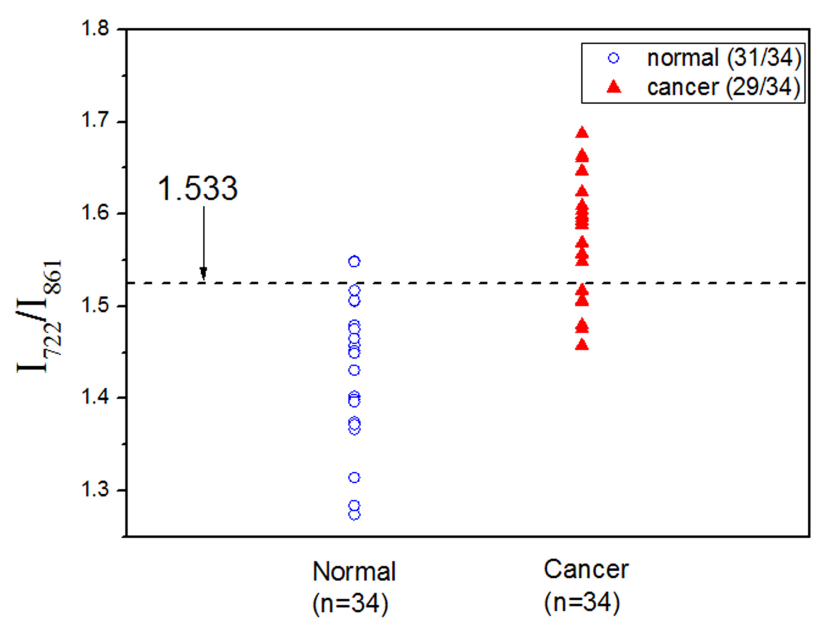

(a)

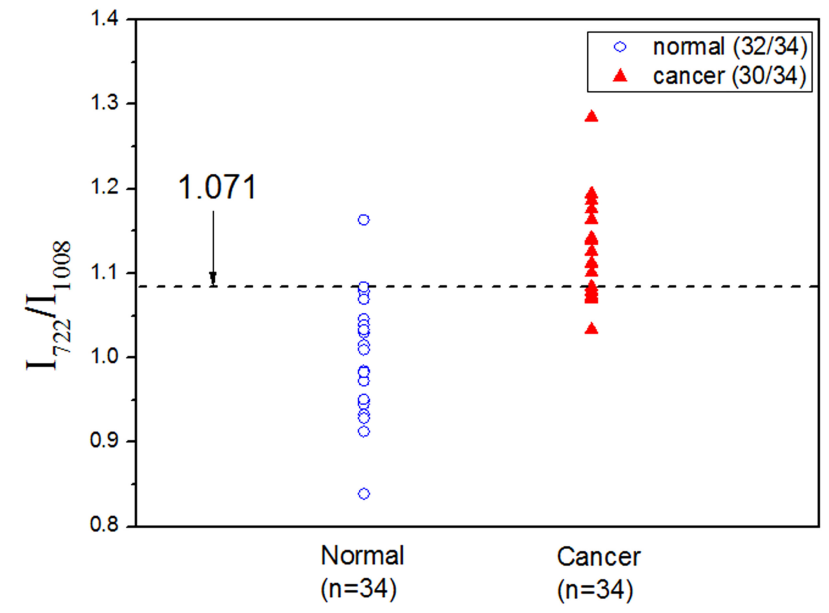

(b)

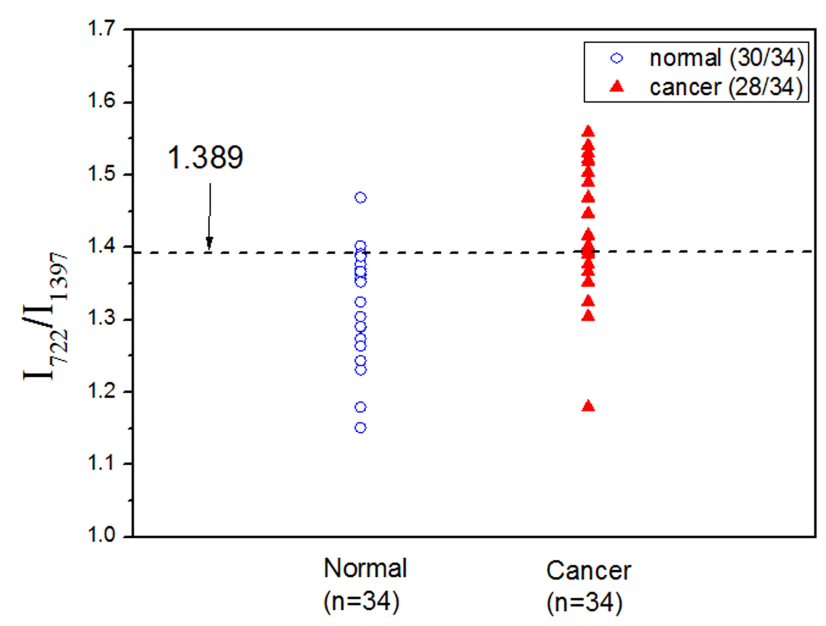

(c)

Fig. 6. Scatter plots of the intensity ratio of the Raman signal at (a) $722 \mathrm{vs} 861 \mathrm{~cm}^{-1}$; (b) $722 \mathrm{vs} 1008 \mathrm{~cm}^{-1}$ and (c) $722 \mathrm{vs} 1395 \mathrm{~cm}^{-1}$ as measured for each sample. The dotted lines $\left(\mathrm{I}_{722} / \mathrm{I}_{861}=1.53 ; \mathrm{I}_{722} / \mathrm{I}_{1008}=1.07\right.$ and $\left.\mathrm{I}_{722} / \mathrm{I}_{1397}=1.39\right)$ as diagnostic threshold detect the gastric cancer with $91.2 \%(31 / 34), 94.1 \%$ (32/34) and 88.2\% (30/34) sensitivity, respectively; 85.3\% (29/34), 88.2\% $(30 / 34)$ and $82.3 \%(28 / 34)$ specificity, respectively.

peaks at $722,861,1008$ and $1397 \mathrm{~cm}^{-1}$ with the largest spectral difference (Fig. 3) and the most statistically significant difference identified through Student's $t$-test (Table 1) were selected for ratio analysis.

Figure 6(a) shows the one-dimensional plot using the $\mathrm{I}_{722} / \mathrm{I}_{861}$ ratio and when the discriminant diagnostic line is 1.53 , the discriminant sensitivity for the serum of cancer patients and healthy volunteers is $91.2 \%(31 / 34)$, and the specificity is $85.3 \%(29 /$ 34). Similarly, with $\mathrm{I}_{722} / \mathrm{I}_{1008}=1.07$ and $\mathrm{I}_{722} /$ $\mathrm{I}_{1397}=1.39$ as diagnosis line, the sensitivities for the serum of cancer patients and healthy volunteers are $94.1 \%(32 / 34)$ and $88.2 \%(30 / 34)$, respectively, and the specificities are $88.2 \%(30 / 34)$ and $82.3 \%(28 /$ 34 ), respectively (see Figs. 6(b) and 6(c)). Therefore, using a single ratio index cannot sufficiently distinguish the serum of gastric cancer from that of healthy volunteers.

Figure 7(a) shows the two-dimensional plot, $\mathrm{I}_{722} /$ $\mathrm{I}_{1008}$ vs $\mathrm{I}_{722} / \mathrm{I}_{861}$, observing that the sensitivity and specificity are $100 \%(34 / 34)$ and $94.1 \%(32 / 34)$, with the accuracy of $97.1 \%(66 / 68)$. In addition, observing the two-dimensional plot, $\mathrm{I}_{722} / \mathrm{I}_{1008}$ vs $\mathrm{I}_{722} / \mathrm{I}_{1397}$, in Fig. $7(\mathrm{~b})$, the sensitivity and specificity are $94.1 \%(32 / 34)$ and $94.1 \%(32 / 34)$, with the accuracy of $94.1 \%(64 / 68)$. It is clear from Fig. 7 that there is slight overlaps between normal and 


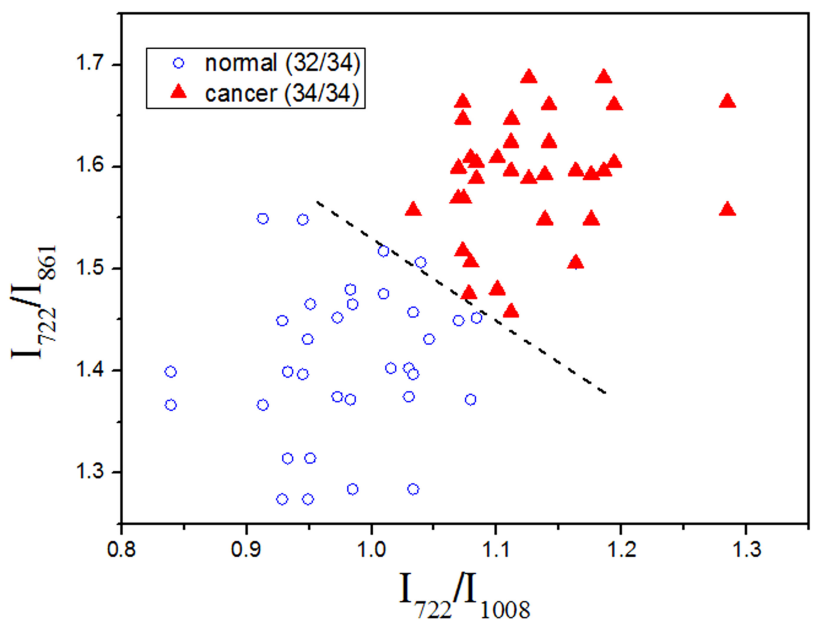

(a)

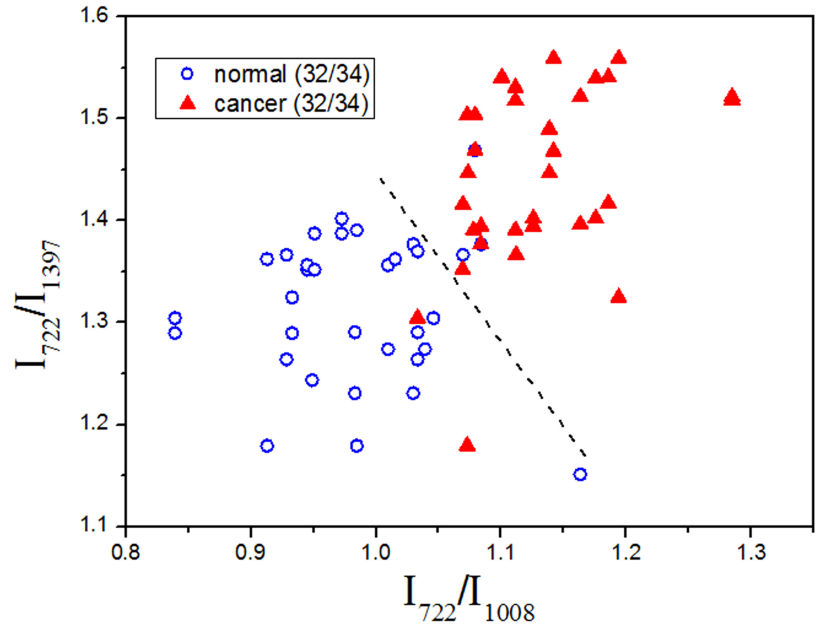

(b)

Fig. 7. Scatter plots of the intensity ratio of serum SERS spectra in gastric cancer and normal group: (a) $\mathrm{I}_{722} / \mathrm{I}_{1008}$ vs $\mathrm{I}_{722} / \mathrm{I}_{861}$; (b) $\mathrm{I}_{722} / \mathrm{I}_{1008}$ vs $\mathrm{I}_{722} / \mathrm{I}_{1397}$.

gastric cancer group, and the healthy volunteers can be easily differentiated from the gastric cancer patients. The spatial distribution of normal and cancer group is relatively concentrated.

The three-dimensional scatter plot based on the intensity ratios of $\mathrm{I}_{722} / \mathrm{I}_{861}, \mathrm{I}_{722} / \mathrm{I}_{1008}$ and $\mathrm{I}_{722} / \mathrm{I}_{1397}$ is shown in Fig. 8, and sensitivity and specificity are $100 \%(34 / 34)$ and $97.4 \%(33 / 34)$, respectively, with the accuracy of $98.5 \%$ (67/68). It is clear from Fig. 8 that the healthy volunteers can be easily differentiated from the patients with gastric cancer, and the space regions of cancer group and the normal group are distributed intensively. This result shows that the SERS spectral data of cancer patients can be

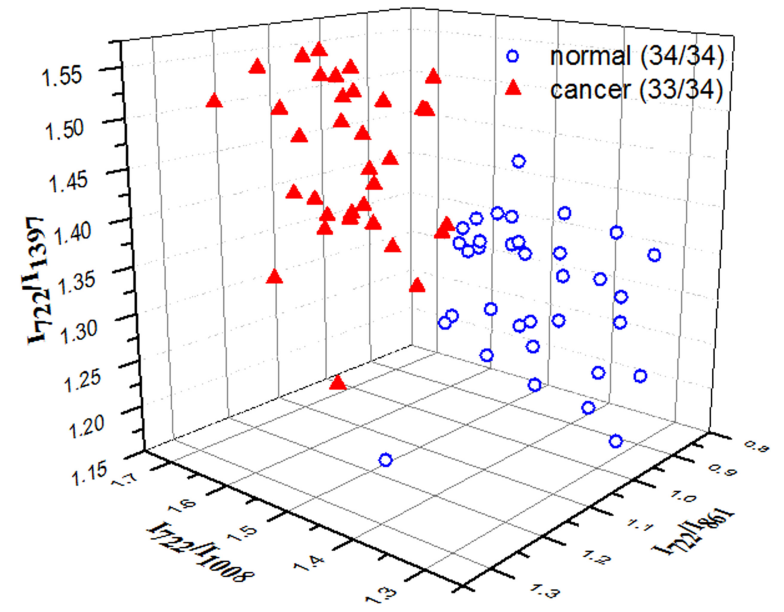

Fig. 8. A three-dimensional scatter plot of intensity ratio of serum SERS spectra in gastric cancer (red triangle) and normal (blue circle) group. directly differentiated from that of healthy volunteers on the basis of the peak intensity ratios with statistically significant differences, and that $\mathrm{I}_{722} /$ $\mathrm{I}_{861}, \mathrm{I}_{722} / \mathrm{I}_{1008}$ and $\mathrm{I}_{722} / \mathrm{I}_{1397}$ can be used as the discriminant parameters for the identification of gastric cancer patients and healthy volunteers.

Comparing and analyzing Figs. 6-8 reveal that classification accuracy can be improved through the selection of multiple intensity ratios. Gastric cancer diagnosis based on SERS spectral data is a multifactor process that involves the extraction of multiple characteristics and not only a single ratio index. The above experiments were based on the intensity ratios of $\mathrm{I}_{722} / \mathrm{I}_{861}, \mathrm{I}_{722} / \mathrm{I}_{1008}$ and $\mathrm{I}_{722} / \mathrm{I}_{1397}$ from SERS spectra, and all these three ratios are significantly higher than that in normal serum. The intensity of SERS peaks at $861 \mathrm{~cm}^{-1}$ (attributable to tryptophan), $1008 \mathrm{~cm}^{-1}$ (attributable to phenylalanine) and $1397 \mathrm{~cm}^{-1}$ (attributable to leucine) weakened in the spectra of cancer serum. In contrast, the intensity of the peak at $722 \mathrm{~cm}^{-1}$ (attributable to adenine) is strengthened. It is indicated that the content and proportion of substances such as adenine in DNA and amino acids have changed in cancer serum, and proves that the canceration is a complex and multifactorial process, which is not only the result of abnormal metabolism of amino acids. This finding is the foundation of gastric cancer diagnosis based on SERS spectra combined with the CRM.

CRM is more suitable than PCA for the discrimination of serum SERS spectral of gastric 
cancer. The peak intensity ratios of $\mathrm{I}_{722} / \mathrm{I}_{861}, \mathrm{I}_{722} /$ $\mathrm{I}_{1008}$ and $\mathrm{I}_{722} / \mathrm{I}_{1397}$ reflect the abnormal metabolism of substances such as amino acids and nucleic acids in the serum of cancer patients, and can be considered as an important fingerprint peak for the early diagnosis and detection of gastric cancer. CRM shows the classified information of samples through the positions of each sample point in the band ratio classification map. Therefore, the classification results of CRM are more intuitive and operational than PCA, moreover, it shortens the time required for classification. With all these advantages, the physiological and pathological changes in the body metabolism can be reflected more efficiently and directly by CRM. The detection of biomolecular changes associated with cancer development is highly important for the early identification and diagnosis of gastric cancer.

\section{Conclusions}

In this paper, with gold nanoparticle as the enhancement base, the serum samples of gastric cancer patients are differentiated from that of healthy volunteers by SERS technique. The Raman intensity of the characteristic peak at $722 \mathrm{~cm}^{-1}$ (attributable to adenine) in serum SERS spectra of gastric cancer patients is stronger than that in serum SERS spectra of healthy volunteers. In contrast, the Raman intensities of peaks at 588, 644, 861, 1008, $1235,1397,1445$ and $1586 \mathrm{~cm}^{-1}$, which are attributable to amino acids, phospholipids, and carbohydrates, in serum SERS spectra of gastric cancer patients are weaker than those in serum SERS spectra of healthy volunteers. Compared with serum samples of healthy volunteers, the protein structure in the cancer serum has changed, and the relative content of adenine increased while the relative content of phospholipid and carbohydrates decreased.

CRM and PCA are performed to analyze and discriminate the serum SERS spectra of healthy volunteers and gastric cancer patients. Results show that the classification performance of CRM is better than that of PCA, and the discrimination sensitivity and specificity are 100\% (34/34) and 97.4\% (33/ $34)$, respectively, with the accuracy of $98.5 \%$ (67/ 68). In contrast to PCA, which requires the use of integrated spectroscopic data, CRM can simply and directly use spectral information by selecting multiple peak intensity ratios. CRM may be applied as a rapid, simple, noninvasive blood detection method for gastric cancer with high accuracy and good popularization potential.

\section{Conflict of Interest}

The authors declare that there are no conflict of interest related to this article.

\section{Acknowledgments}

This work was supported by the Natural Science Foundation of Guangdong Province, China (2018 A0303131000), the project of Academician workstation of Guangdong Province, China (2014B0 90905001), the Fundamental Research Funds for the Central Universities, China (21617406) and the key project of Scientific and Technological projects of Guang Zhou, China (201604040007, 201604020168). We also gratefully acknowledge many of our colleagues for their stimulating discussions in this field.

\section{Ethics}

Serum samples were selected randomly collected from physical examination respondents of different departments of the First Affiliated Hospital of Jinan University. The research described in this paper was performed with full ethical approval and the patient was informed of the situation.

\section{References}

1. Y. Nie, K. Wu, J. Yu, Q. Liang, X. Cai, Y. Shang, J. Zhou, L. Pan, L. Sun, J. Fang, "A global burden of gastric cancer: The major impact of China," Expert. Rev. Gastroent. 11(7), 651-661 (2017).

2. Z. Huang, S. K. Teh, W. Zheng, K. Lin, K. Y. Ho, M. Teh, K. G. Yeoh, "In vivo detection of epithelial neoplasia in the stomach using image-guided Raman endoscopy," Biosens. Bioelectron. 26(2), 383-389 (2010).

3. S. K. Teh, W. Zheng, K. Y. Ho, M. Teh, K. G. Yeoh, "Near-infrared Raman spectroscopy for early diagnosis and typing of adenocarcinoma in the stomach," Brit. J. Surg. 97(4), 550-557 (2010).

4. S. Duraipandian, M. S. Bergholt, W. Zheng, K. Y. Ho, M. Teh, K. G. Yeoh, J. B. Y. So, A. Shabbir, Z. Huang, "Real-time Raman spectroscopy for in vivo, online gastric cancer diagnosis during clinical endoscopic examination," J. Biomed. Opt. 17(8), 081418 (2012). 
5. J. T. Motz, S. J. Gandhi, O. R. Scepanovic, A. S. Haka, J. R. Kramer, R. R. Dasari, M. S. Feld, "Real-time Raman system for in vivo disease diagnosis," J. Biomed. Opt. 10(3), 031113 (2005).

6. H. Zeng, J. Zhao, M. Short, D. I. Mclean, S. Lam, A. Mcwilliams, H. Lui, "Raman spectroscopy for in vivo tissue analysis and diagnosis, from instrument development to clinical applications," $J$. Innov. Opt. Heal. Sci. 1(1), 95-106 (2008).

7. S. K. Teh, W. Zheng, D. P. Lau, Z. Huang, "Spectroscopic diagnosis of laryngeal carcinoma using near-infrared Raman spectroscopy and random recursive partitioning ensemble techniques," Analyst 134, 1232-1239 (2009).

8. M. S. Bergholt, W. Zheng, K. Y. Ho, M. Teh, K. G. Yeoh, J. B. Y. So, A. Shabbir, Z. Huang, "Fiberoptic Raman spectroscopy probes gastric carcinogenesis in vivo at endoscopy," J. Biophotonics. 6(1), 49-59 (2013).

9. A. Downes, A. Elfick, "Raman spectroscopy and related techniques in biomedicine," Sensors. 10(3), 1871-1889 (2010).

10. M. Vendrell, K. K. Maiti, K. Dhaliwal, Y. T. Chang, "Surface-enhanced Raman scattering in cancer detection and imaging," Trends. Biotechnol. 31(4), 249-257 (2013).

11. H. J. Butler, S. W. Fogarty, J. G. Kerns, P. L. MartinHirsch, "Gold nanoparticles as a substrate in bioanalytical near-infrared surface-enhanced Raman spectroscopy," Analyst 140(9), 3090-3097 (2015).

12. Y. Wang, J. Irudayaraj, "Surface-enhanced Raman spectroscopy at single-molecule scale and its implications in biology," Philos. T. R. Soc. B 368(1161), 20120026 (2013).

13. P. H. Hsua, H. K. Chianga, "Raman spectroscopy forquantitative measurement of lactic acid atphysiological concentration in human serum," J. Raman. Spectroscopy. 41(12), 1610-1614 (2010).

14. S. K. Teh, W. Zheng, K. Y. Ho, M. Teh, K. G. Yeoh, Z. Huang, "Diagnosis of gastric cancer using nearinfrared Raman spectroscopy and classification and regression tree techniques," J. Biomed. Opt. 13(3), 034013 (2008).

15. R. Xiao, X. Zhang, Z. Rong, B. Xiu, X. Yang, C. Wang, W. Hao, Q. Zhang, Z. Liu, C. Duanb, K. Zhao, X. Guo, Y. Fan, Y. Zhao, H. Johnson, Y. Huang, X. Feng, X. Xu, H. Zhang, S. Wang, "Noninvasive detection of hepatocellular carcinoma serum metabolic profile through surface-enhanced Raman spectroscopy," Nanomedicine 12(8), 24752484 (2016).

16. J. D. Guingab, B. Lauly, B. W. Smith, N. Omenetto, J. D. Winefordner, "Winefordner. Stability of silver colloids as substrate for surface enhanced Raman spectroscopy detection of dipicolinic acid," Talanta 74(2), 271-274 (2007).

17. M. A. Cipriano, M. Areia, P. Amaro, M. DinisRibeiro, M. A. Cipriano, C. Marinho, A. Costa-Pereira, C. Lopes, L. Moreira-Dias, J. M. Romaozinho, H. Gouveia, D. Freitas, M. C. Leitao, "External validation of a classification for methylene blue magnification chromoendoscopy in premalignant gastric lesions," Gastrointest. Endosc. 67(7), 1011-1018 (2008).

18. S. Cervo, E. Mansutti, G. D. Mistro, R. Spizzo, A. Colombatti, A. Steffan, V. Sergo, A. Bonifacio, "SERS analysis of serum for detection of early and locally advanced breast cancer," Anal. Bioanal. Chem. 407(24), 7503-7509 (2015).

19. H. Chon, S. Lee, S. W. Son, C. H. Oh, J. Choo, "Highly sensitive immunoassay of lung cancer marker carcinoembryonic antigen using surface-enhanced raman scattering of hollow gold nanospheres," Anal. Chem. 81(8), 3029-3034 (2009).

20. S. Lee, H. Chon, J. Lee, J. Ko, B. H. Chung, D. W. Lim, J. Choo, "Rapid and sensitive phenotypic marker detectionon breast cancer cells using surfaceenhanced Raman scattering (SERS) imaging," Biosens. Bioelectron. 51, 238-243 (2014).

21. X. Li, T. Yang, S. Li, L. Jin, D. Wang, D. Guan, J. Ding, "Noninvasive liver diseases detection based on serum surface enhanced Raman spectroscopy and statistical analysis," Opt. Exp. 23(14), 18361-18372 (2015).

22. D. Rohleder, W. Kiefer, W. Petrich, "Quantitative analysis of serum and serum ultrafiltrate by means of Raman spectroscopy," Analyst 129(10), 906-911 (2004).

23. S. Fenga, R. Chen, J. Lin, J. Pan, Y. Wu, Y. Li, J. Chen, H. Zeng, "Gastric cancer detection based on blood plasma surface-enhanced Raman spectroscopy excited by polarized laser light," Biosens. Bioelectron. 26(7), 3167-3174 (2011).

24. E. Vargas-Obieta, J. C. Martínez-Espinosa, B. E. Martínez-Zérega, L. F. Jave-Suárez, A. AguilarLemarroy, J. L. González-Solís, "Breast cancer detection based on serum sample surface enhanced Raman spectroscopy," Lasers Med. Sci. 31(7), 1317-1324 (2016).

25. S. A. Sánchez-Rojo, B. E. Martínez-Zérega, E. F. Velázquez-Pedroza, J. C. Martínez-Espinosa, L. A. Torres-González, A. Aguilar-Lemarroy, L. F. JaveSuárez, P. Palomares-Anda, J. L. González-Solís, "Cervical cancer detection based on serum sample surface enhanced Raman," Rev. Mex. Fis. 62(3), 213-218 (2016).

26. D. Lin, S. Y. Feng, J. J. Pan, Y. P. Chen, J. Q. Lin, G. N. Chen, S. S. Xie, H. S. Zeng, R. Chen, 
"Colorectal cancer detection by gold nanoparticle based surface-enhanced Raman spectroscopy of blood serum and statistical analysis," Opt. Exp. 19 (14), 13565-13577 (2011).

27. H. Xiong, Z. Guo, H. Zhong, Y. Ji, "Monitoring the penetration and accumulation of gold nanoparticles in rat skin ex vivo using surface-enhanced Raman scattering spectroscopy," J. Innov. Opt. Heal. Sci. 9(5), 1650026-1-11 (2016).

28. S. Feng, D. Lin, J. Lin, B. Li, Z. Huang, G. Chen, W. Zhang, L. Wang, J. Pan, R. Chen, H. Zeng, "Blood plasma surface-enhanced Raman spectroscopy for non-invasive optical detection of cervical cancer," Analyst 138(14), 3967-3974 (2013).

29. P. Rekha, P. Aruna, G. Bharanidharan, D. Koteeswaran, M. Baludavidc, S. Ganesan, "Near infrared Raman spectroscopic characterization of blood plasma of normal, oral premalignant and malignant conditions-a pilot study," J. Raman. Spectrosc. 46(9), 735-743 (2015).

30. S. X. Li, Y. J. Zhang, Q. Y. Zeng, L. F. Li, Z. Y. Guo, Z. M. Liu, H. L. Xiong, S. H. Liu, "Potential of cancer screening with serum surface-enhanced Raman spectroscopy and a support vector machine," Laser. Phys. Lett. 11(6), 1-7 (2014).

31. S. K. Teh, W. Zheng, K. Y. Ho, M. Teh, K. G. Yeoh, Z. Huang, "Diagnostic potential of near-infrared Raman spectroscopy in the stomach: Differentiating dysplasia from normal tissue," Brit. J. Cancer 98(2), 457-465 (2008).

32. J. L. Pichardo-Molina, C. Frausto-Reyes, O. Barbosa-García, R. Huerta-Franco, J. L. GonzálezTrujillo, C. A. Ramírez-Alvarado, G. GutiérrezJuárez, C. Medina-Gutiérrez, "Raman spectroscopy and multivariate analysis of serum samples from breast cancer patients," Laser Med. Sci. 22(4), 229-236 (2007).

33. U. Utzinger, D. L. Heintzelman, A. MahadevanJansen, A. Malpica, M. Follen, R. Richards-Kortum, "Near-infrared raman spectroscopy for in vivo detection of cervical precancers," Appl. Spectrosc. 55(8), 955-959 (2001). 